\title{
Spontaneous uretero-sigmoid fistula secondary to calculus
}

\author{
Ines Marzouk, MD;1 Makram Moussa, MD;' Lotfi Saadallah, MD; ${ }^{3}$ Sami Bouchoucha, MD;2 \\ Lotfi Hendaoui, $M D^{1}$
}

'Department of Diagnostic and Interventional Radiology, University Hospital of Mongi Slim, La Marsa, Tunisia, ${ }^{2}$ Department of Surgery, University Hospital of Bizerta, Tunisia; ${ }^{3}$ Department of Urology, University Hospital of Bizerta, Tunisia

Cite as: Can Urol Assoc J 2016;10(11-12):E401-3. http://dx.doi.org/10.5489/cuai.3402 Published online November 10, 2016.

\section{Abstract}

A 25-year-old man was referred to the urology department after a subacute history of left back pain, burning micturition associated with pneumaturia and fecaluria. Ultrasonography was performed showing hydronephrosis, and plain film radiography demonstrated a long vertical left pelvic calculi. Uro-computed tomography (CT) combined with a water enema CT showed a $10 \mathrm{~cm}$ long calculus with the cranial extremity fistulating the sigmoidal wall. Surgical treatment included left nephroureterectomy and sigmoidectomy with a colorectal anastomosis. Postoperative course was uneventful.

\section{Case report}

A 25-year-old man without any notable personal or familial medical history consulted in the urology department. A subacute clinical history had started one week before with left back pain and burning micturition. He had progressively observed pneumaturia and fecaluria. Clinical outcomes were non-specific; the patient was febrile at $38{ }^{\circ} \mathrm{C}$ and the left lumbar region was painful.

Investigations started with laboratory tests and radiological examinations. C-reactive protein was elevated at $50 \mathrm{mg} / \mathrm{l}$. Blood creatinine and leucocyte count were normal. Plain film showed a long stick-shaped vertical calcification in the left pelvic area (Fig. 1).

Ultrasonography found a dilation of the urinary tract upstream of the calculi. Computed tomography (CT) scan was performed. Protocol technique started with an unenhanced CT phase; after a water enema, a multiphase CT urography was performed with the purposes of doing a presurgical assessment and exploring the left renal function. On CT scans, the $10 \mathrm{~cm}$ long calculus was localized in the lumbar ureter protruding in the colonic lumen; the density was 835 Hounsfield units (Fig. 2).

A fat standing was noticed around the fistula without any liquid effusion. Multiple millimetric caliceal calculi were observed as well. After contrast media administration, non-excretion was noticed in the left kidney. Endoscopic examination of the colon was not tolerated by the patient.

A congenital megaureter was suspected as the cause of calculi development seeing as the patient was young, the ureter was very dilated, and hydronephrosis was present. As the patient was living in an endemic country, urinary tuberculosis was also not excluded as a possible cause.

Surgery was undertaken by the visceral surgeon and the urologist. Intraoperative findings were ureteral dilation and a fistula between the sigmoid and the ureter (Fig. 3). The first step of the procedure was a left nephroureterectomy. Then, a partial sigmoidectomy was performed, followed by a colorectal anastomosis. The patient was treated with triple antibiotherapy.

Surgical courses were uneventful. Pain and fever regressed within 24 hours. The cytobacterioligic urine examination and culture did not identify any Koch's bacilli, not did the histopathological examination of the renal specimen.

\section{Discussion}

Spontaneous fistulae between the ureter and the colon are rare. This is only the third reported case of ureterosigmoid spontaneous fistula caused by a calculus in the literature. ${ }^{1,2}$ To our knowledge, no other case have described the combination of water enema and CT urography for the diagnosis of ureterocolic fistula.

Spontaneous ureterocolic fistulae usually occur secondary to tuberculosis, Crohn's disease, pelvic malignancies, and renal calculi. ${ }^{3-6}$ Prior to effective therapy, tuberculosis was the most common etiology for these fistulae. ${ }^{7}$ Most spontaneous ureterocolic fistulae are now caused by urinary calculi. Extracorporeal shockwave lithotripsy and impacted calculi have also been reported as rare causes of ureterocolic fistula. ${ }^{8,9}$ Ureterocolic fistulae can also occur after pelvic surgery with iatrogenic trauma, renal transplantation, or radiotherapy. ${ }^{7}$

The most evocative clinical signs are pneumaturia, which is the passage of gas in the urine during or after urination, and fecaluria, the presence of fecal particles in the urine. Other non-specific signs, such as abdominal pain, fever, or pyuria, can be associated. Urinalysis and urine culture can 


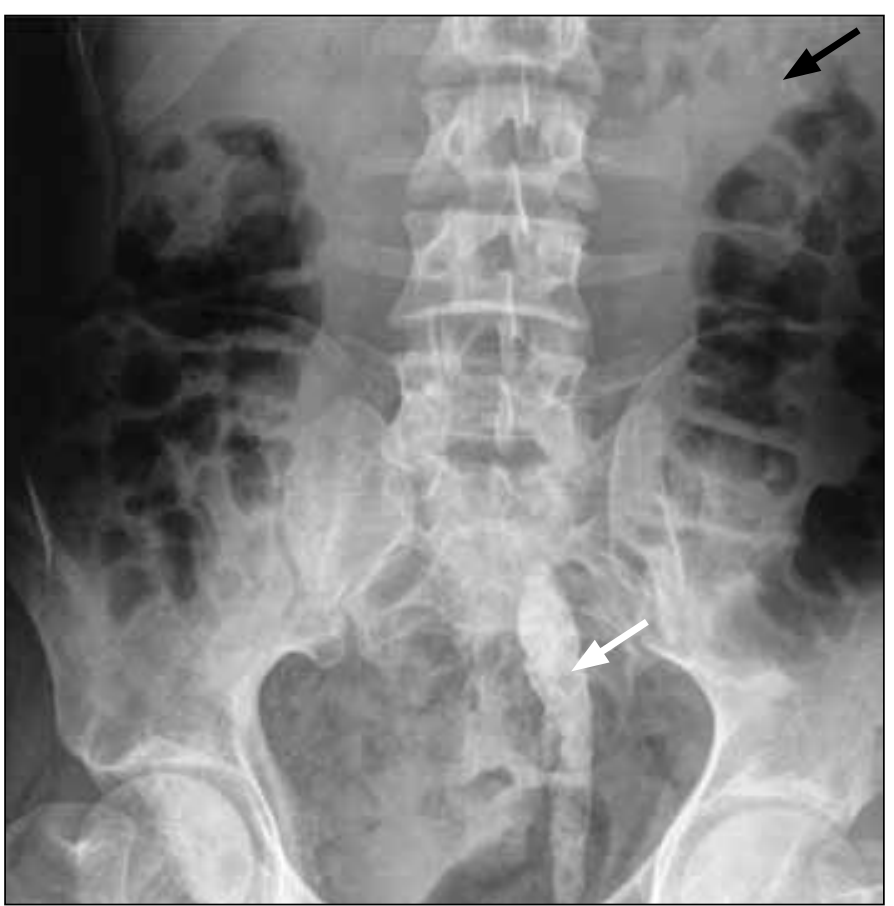

Fig. 1. Plain film radiography showing a long stick-shaped vertical calcification in the left pelvic area (white arrow). Notice the other calcified opacities upper in the left kidney (black arrow).

reveal a urinary infection.

Ultrasonography and plain film radiography are the first examinations to perform when faced with non-specific urinary signs. Diagnosis of enterourinary fistula is based on opacification of the urinary tract with an abnormal passage of contrast media from the ureter to the colon. The sensitivity of depiction of fistulae was made higher with the multidetector $\mathrm{CT}$ scans and magnetic resonance imaging
(MRI). CT urography has progressively replaced the excretory urography and the retrograde pyelography, allowing a better space resolution. Direct sign of fistula on $\mathrm{CT}$ is rarely revealed on the delayed (excretory) phase after contrast media administration. ${ }^{10}$ The use of multiplanar or curved planar reformatting can improve the diagnosis. Indirect signs are the presence of gas in the urinary tract and ureter wall thickening. ${ }^{2,10}$

The etiology of the fistula can also be provided by CT scan. In case of diverticular disease, multiple diverticuli are described along the left colon with a fat standing in front of the incriminated diverticulum. Abnormal anatomy, enteric wall thickening, and measure of the density of the calculus are also valuable pieces of information provided by the $\mathrm{CT}$ scan.

The management of the ureterocolic fistulae is variable. Minimally invasive radiological techniques with percutaneous nephrostomy and double J stent are increasingly performed. ${ }^{11}$ Treatment of an ureteroenteric fistula involves ureterolysis and possible bowel resection. Nephrectomy is the standard surgery for a non-functioning kidney, ${ }^{7}$ as was the case with our patient.

\section{Conclusion}

Spontaneous ureterocolic fistula caused by a calculus is a rare entity. The diagnostic challenge is based on an appropriate imaging technique. In this case, non-invasive imaging association of a water enema with multidetector CT urography helped view the fistula tract.

\section{Competing interests: The authors report no competing personal or financial interests.}

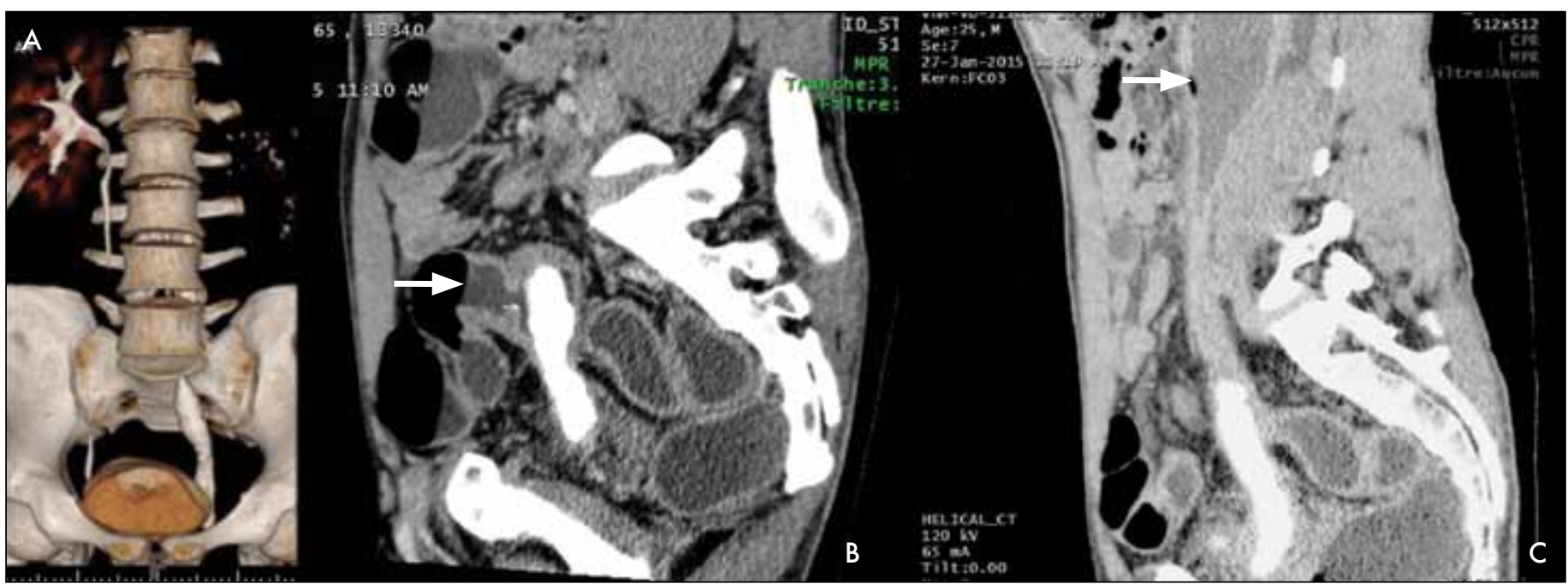

Fig. 2. Uro-computed tomography (CT) scans with water enema; $(\boldsymbol{A})$ CT volume rendering scan at the delayed phase: non-functioning left kidney with multiple caliceal calculi; (B) multiplanar reformat CT scan showing the calculus presenting its cranial extremity in the sigmoidal lumen (arrow); (C) CT curved planar reformat in the axis of the left ureter showing a gas bubble (arrow) in the dilated ureter. 


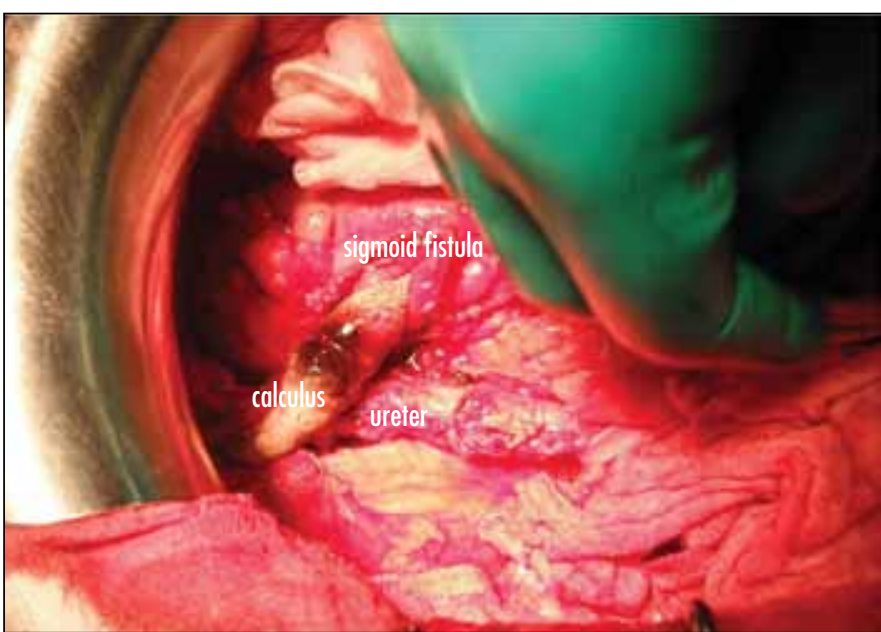

Fig. 3. Intraoperative view after nephrectomy and before sigmoidectomy. Colonic fistula is well-identified with the calculus.

This paper has been peer-reviewed.

\section{References}

1. Lee WK, Chang SD, Roche CJ, et al. Spontaneous ureterocolic fistula secondary to calculous pyohydroureteronephrosis. Br J Radiol 2005;78:954-5. http://dx.doi.org/10.1259/bir/53711174

2. Earlam MSS. Migration of a ureteric calculus to the bladder via a ureterocolic and a vesicocolic fistula. Aust N Z I Surg 1977;47:519-20. http://dx.doi.org/10.1111/i.1445-2197.1977.tb04339.x

3. Pai A, Coralic J, Park JJ, et al. Spontaneous ureterocolic fistula secondary to diverticulitis: Report of a case and review of literature. Int J Colorectal Dis 2013;29:417-8. http://dx.doi.org/10.1007/ s00384-013-1813-7

4. Wc C, Sr P, Rw G. Spontaneous ureterocolic fistula: A rare complication of colonic diverticular disease. Am Surg 1994;60:832-5.

5. Conde Santos G, Gri-ó Garreta J, Bielsa Gali 0, et al. Ureterocolonic fistula in non-functioning ureter. Arch Esp Urol 2001;54:1126-9.

6. Virseda JA, Vera JA, Recio R, et al. Spontaneous pneumopyelography: Ureterocolic fistula caused by rectosigmoid carcinoma. Arch Esp Urol 1981;34:289-96.

7. Chen S-H, Wu W-J, Chen H-W et al. Ureterocolonic fistula formation 12 years after a nephrectomy for renal tuberculosis with a staghorn stone. Urol Sci 2013;24:69-71. http://dx.doi.org/10.1016/i. urols.2013.04.007

8. Patil KP, Shetty SD, Anandan N et al. Ureterocolic fistula due to impacted ureteric stone. $\mathrm{Br} J$ Urol 1992;70:332-3. http://dx.doi.org/10.1111/i.1464-410X.1992.tb15747.x

9. Flood HD, Jones B, Grainger R. Ureterocolic fistula: A unique complication of extracorporeal shockwave lithotripsy. J Urol 1992;147:122-4.

10. Yu NC, Raman SS, Patel M, et al. Fistulas of the genitourinary tract: A radiologic review. RadioGraphics 2004;24:1331-52. http://dx.doi.org/10.1148/rg.245035219

11. Lang EK, Allaei A, Robinson L, et al. Minimally invasive radiologic techniques in the treatment of ureteroenteric fistulas. Diagn Interv Imaging 2015;96:1153-60. http://dx.doi.org/10.1016/iddiii.2015.06.010

Correspondence: Dr. Ines Marzouk, Department of Diagnostic and Interventional Radiology University Hospital of Mongi Slim, La Marsa, Tunisia; marzouk.ines@gmail.com 